\title{
ORIGINAL RESEARCH \\ Parasellar T2 Dark Sign on MR Imaging in Patients with Lymphocytic Hypophysitis
}

Y. Nakata

N. Sato

T. Masumoto

H. Mori

H. Akai

H. Nobusawa

Y. Adachi

H. Oba

K. Ohtomo

BACKGROUND AND PURPOSE: MR imaging findings of LYH and pituitary adenomas are similar, but the therapeutic strategies are completely different. The purpose of this study was to evaluate sellar and parasellar MR imaging findings in patients with both diseases, as well as characteristic clinical findings.

MATERIALS AND METHODS: Clinical findings, including endocrinologic study and MR images of 20 patients with LYH and 22 patients with pituitary adenoma, were retrospectively reviewed. We evaluated the MR images in relation to the following: 1) the PPHI on T1-weighted images, 2) thickened stalk (>3.5 mm), 3) pituitary symmetry, 4) pituitary enhancement pattern, 5) a dural tail, and 6) parasellar signal intensity on T2- and T1-weighted images.

RESULTS: Between patients with LYH and those with pituitary adenoma, a significant difference was identified for the number of patients with loss of PPHI, thickened stalk, pituitary symmetry, homogeneous enhancement, and parasellar dark signal intensity on T2-weighted images by statistical analysis (Fisher exact probability test, $P<.05$ ). Among them, only parasellar dark signal intensity on T2weighted images had no false-positive cases.

CONCLUSIONS: The parasellar T2 dark sign can be a specific finding used to distinguish pituitary adenoma from $\mathrm{LYH}$.

\begin{abstract}
ABBREVIATIONS: $\mathrm{ACTH}=$ adrenocorticotrophic hormone; $\mathrm{AL}=$ anterior lobe; $\mathrm{DI}=$ diabetes insipidus; $\mathrm{Dp}=$ desmopressin; $\mathrm{FSH}=$ follicle-stimulating hormone; $\mathrm{Gc}=$ glucocorticoid; $\mathrm{GH}=$ growth hormone; $\mathrm{GHs}=\mathrm{GH}$-secreting; ICA = internal carotid artery; IgG4 = immunoglobulin 4; Iso = isointense; $\mathrm{L}=$ left; $\mathrm{LH}=$ luteinizing hormone; $\mathrm{Lt}=$ levothyroxine; $\mathrm{LYH}=$ lymphocytic hypophysitis; MRI = MR imaging; Non- $f=$ nonfunctioning; $P A=$ pituitary adenoma; Pan-hypo = panhypopituitarism; Partial-hyper = partial hyperpituitarism; Partial-hypo = partial hypopituitarism; $\mathrm{PL}=$ posterior lobe; $\mathrm{PPHI}=$ posterior pituitary $\mathrm{T} 1$ high intensity; $\mathrm{PRL}=$ prolactin; $\mathrm{PRLS}=$ PRL-secreting; $R$ = right; synd = syndrome; $T 1 \mathrm{WI}=\mathrm{T} 1$-weighted imaging; $\mathrm{T} 2 \mathrm{WI}=\mathrm{T} 2$-weighted imaging; $\mathrm{Ts}=$ testosterone; $\mathrm{TSH}=$ thyroid-stimulating hormone
\end{abstract}

$\mathbf{L}$ YH is a rare inflammatory disease of the pituitary gland. This condition is characterized by lymphocytic infiltration and eventual destruction of the pituitary tissue accompanied by various degrees of pituitary dysfunction. This disease is now known to affect both pituitary lobes, at all ages and in both sexes. ${ }^{1-6}$ An autoimmune pathogenesis is suggested by several histopathologic, laboratory, and clinical findings. ${ }^{7}$

LYH is often misdiagnosed because its clinical and radiologic features mimic tumors in the sellar and parasellar region. ${ }^{1}$ The main diagnostic issue is that $\mathrm{LYH}$ is a relatively rare disease, and its imaging findings are not well-recognized in contrast to those of overwhelmingly more common pituitary tumors, pituitary adenomas. ${ }^{8}$ Distinguishing LYH from pitu-

\section{Received March 9, 2010; accepted after revision May 10}

From the Department of Radiology (Y.N., N.S.), National Center Hospital of Neurology and Psychiatry, Tokyo, Japan; Department of Radiology (Y.N., H.M., H.A., K.O.), Faculty of Medicine, University of Tokyo, Tokyo, Japan; Department of Diagnostic Radiology (N.S.), Faculty of Medicine, University of Gumma, Maebashi, Japan; Department of Radiology (T.M.), Faculty of Medicine, University of Tsukuba, Tsukuba, Japan; Department of Radiology (H.N.), Faculty of Medicine, Showa University, Tokyo, Japan; Department of Radiology (Y.A.), University of California, San Francisco, San Francisco, California; Department of Radiology (Y.A., H.O.), Faculty of Medicine, Teikyo University, Tokyo, Japan; and Department of Radiology (H.O.), Showa General Hospital, Tokyo, Japan.

Please address correspondence to Noriko Sato, MD, PhD, Department of Radiology, National Center Hospital of Neurology and Psychiatry, 4-1-1 Ogawahigashi, Kodaira, Tokyo 187-8511, Japan; e-mail address: snoriko@ncnp.go.jp

Eindicates article with supplemental on-line tables.

DOl 10.3174/ajnr.A2201 itary adenomas is very important because different therapeutic strategies are used to treat the 2 diseases. The treatment of LYH remains controversial, though conservative management with close clinical observation has been advocated on the basis of its often benign transient course. ${ }^{9,10}$ The correct diagnosis of LYH contributes to avoiding needless surgery, which is invasive and sometimes results in endocrine dysfunction. However, distinguishing LYH from pituitary adenomas can be difficult. Leung et $\mathrm{al}^{9}$ reported that even with MR imaging studies, approximately $40 \%$ of the cases are misdiagnosed preoperatively as pituitary adenomas. MR imaging findings in patients with LYH and pituitary adenoma were reported in some recent articles. ${ }^{2,3,8,11-14}$ However, most previous reports of MR imaging findings of LYH were case reports and/or reviews of the literature.

Recently, we experienced some cases of LYH, which showed dark-signal-intensity areas on T2-weighted images around the pituitary gland and in the cavernous sinus. To the best of our knowledge, no studies have reported such signalintensity abnormalities. We hypothesized that dark-signalintensity areas on T2-weighted images around the pituitary gland and in the cavernous sinus were characteristic in patients with LYH and were useful for distinguishing pituitary adenoma from LYH.

The present study was retrospectively performed to review a series of clinical and MR imaging findings in patients with LYH, including signal intensity around the pituitary gland. As 
a control study, we also evaluated clinical and MR imaging findings in patients with pituitary adenoma for comparison.

\section{Materials and Methods}

\section{Patients}

We retrospectively reviewed the MR imaging findings in 20 patients with LYH who were selected by review of clinical records. Our local ethics committee did not require its approval or informed consent for this retrospective review. A retrospective review of clinical records in 6 hospitals from 1987 to 2009 revealed 24 patients who were clinically diagnosed as LYH. Two patients who had a history of sarcoidosis or pachymeningitis, and 2 patients whose initial MR images had not been available were excluded from the study. As a consequence, 20 patients with LYH were enrolled. Ten were males and 10 were females, from 9 to 72 years of age, with a mean age of $46.0 \pm 19.5$ years (On-line Table 1). Four of 20 patients had histologically proved disease by transsphenoidal biopsy. The remaining 16 patients were diagnosed on the basis of clinical and endocrinologic studies, MR imaging findings, response to steroid therapy, and natural clinical course. ${ }^{4,7}$ Five patients had a history of autoimmune diseases, such as IgG4related disorders, rheumatoid arthritis, and bullous pemphigoid. One patient was pregnant.

We also reviewed the clinical and MR imaging findings in 22 patients with pituitary adenoma. A retrospective review of clinical records in 1 hospital from 2002 to 2009 revealed 52 patients who were treated with transsphenoidal resection and had a proved pathologic diagnosis of pituitary adenoma. Sixteen patients who had recurrent pituitary adenomas and underwent a second operation, 11 patients whose pathologies did not differentiate between functioning and nonfunctioning adenoma because immunostaining was not performed, 2 patients whose initial MR images had not been available, and 1 patient who received irradiation due to malignancy were excluded from the study. As a consequence, 22 patients with pituitary adenoma were enrolled. Ten were men and 12 were women, and they ranged in age from 25 to 73 years, with a mean age of $50.3 \pm 15.8$ years (On-line Table 2). All patients were treated with transsphenoidal resection, and their pathologies were proved.

In all patients with LYH and pituitary adenoma, the plasma concentrations of ACTH, GH, PRL, LH, FSH, TSH, cortisol, free T4, and testosterone were measured. Provocative tests were performed as follows: for TSH and PRL, after a bolus injection of $500 \mathrm{mg}$ of TSHreleasing hormone; for $\mathrm{LH}$ and $\mathrm{FSH}, 100 \mathrm{mg}$ of gonadotropin-releasing hormone; for $\mathrm{GH}, 100 \mathrm{mg}$ of $\mathrm{GH}$-releasing hormone or $0.1 \mathrm{U}$ of regular insulin per kilogram of body weight; and for cortisol, $0.25 \mathrm{mg}$ of ACTH or $0.1 \mathrm{U}$ of regular insulin per kilogram of body weight. Multiple blood samples were collected to measure plasma hormone concentrations before and for up to 120 minutes after the injections. In patients with LYH except for cases 2, 8, and 13, plasma vasopressin concentrations were determined by radioimmunoassay, and plasma and urinary osmolarities were measured before and after 4-8 hours of water deprivation. In cases $9,11,21$, and 22 of patients with pituitary adenoma, hormonal examination was done for the posterior lobe by using the same method.

\section{MR Imaging Acquisition}

All patients with LYH and pituitary adenoma underwent MR imaging of the sella turcica with $1.5 \mathrm{~T}$ superconductive units. Patients with LYH underwent MR imaging with 6 units in 6 hospitals, and patients with pituitary adenoma underwent $1 \mathrm{MR}$ imaging in 1 hospital. T1-weighted images were acquired in the coronal and sagittal planes with the following parameters: TR/TE/NEX $=300-$ $660 / 7.9-23 \mathrm{~ms} / 3-4$, a $192 \times 256$ to $512 \times 512$ matrix, a 14 - to $20-\mathrm{cm}$ FOV, a $3-\mathrm{mm}$ section thickness, and a $0.0-$ to $0.6-\mathrm{mm}$ intersection gap. T2-weighted sagittal or coronal images were obtained with the following parameters: TR/TE/NEX $=2000-3500 /$ $80-120 \mathrm{~ms} / 3-4$, a $192 \times 256$ to $512 \times 512$ matrix, a $14-$ to $20-\mathrm{cm}$ FOV, a $3-\mathrm{mm}$ section thickness, and a 0.0 - to $0.6-\mathrm{mm}$ intersection gap. T1-weighted images after an intravenous injection of contrast medium $(0.1 \mathrm{mmol} / \mathrm{kg}$ of body weight $)$ were also acquired, except for case 8 , in patients with $\mathrm{LYH}$, in the coronal and sagittal planes with the same parameters as those of the T1-weighted images. All patients with LYH had 1-6 follow-up MR imaging studies performed during a period ranging from 1 month to 8 years.

Two neuroradiologists (Y.N., N.S.) who did not know the patients' clinical information evaluated the MR imaging findings of the patients with LYH and pituitary adenoma. In several recent articles, some findings were reported to be useful for distinguishing pituitary adenoma from LYH, such as precontrast homogeneous signal intensity, intact sellar floor, suprasellar extension, loss of PPHI, stalk thickening, mass symmetry, homogeneous enhancement, and adjacent dural enhancement (the so-called dural tail). ${ }^{2,3,8,11-14}$ In this study, evaluation of the precontrast signal intensity was excluded because pituitary adenomas often show complicated signal intensities due to hemorrhagic or cystic changes, and it is a not suitable factor for making a simple assessment. The findings of destruction of the sellar floor and extension to the sphenoid sinus were also excluded because it was obvious that these findings indicate neoplasm. The finding of suprasellar extension was also excluded because it depends on the size of pituitary lesions, and pituitary adenomas vary in size from microadenoma to macroadenoma.

We, therefore, selected the following MR findings as assessment factors: 1) PPHI on T1-weighted images, 2) thickened stalk, 3) pituitary symmetry, 4) pituitary enhancement pattern, 5) the dural tail, and 6) parasellar signal intensity on T2- and T1-weighted images. PPHI was classified as either "identified" or "not identified." ${ }^{15}$ If the diameter of the pituitary stalk exceeded $3.5 \mathrm{~mm}$ at the level of the median eminence of the hypothalamus, the stalk was considered "thick." ${ }^{16}$ Pituitary symmetry was classified as either "symmetric" or "asymmetric." 15 The enhancement pattern was classified as either "homogeneous" or "heterogeneous." The dural tail was classified as either "identified" or "not identified." Parasellar signal intensity on T2- and T1-weighted images was classified as either "dark," "low," "iso," or "high" intensity. On T2-weighted images, "dark" was isointense with bone cortex; "low" was isointense with white matter; "iso" was isointense with gray matter, and "high" was hyperintense with gray matter. On T1-weighted images, "dark" was isointense with bone cortex; "low" was isointense with CSF, "iso" was isointense with gray matter, and "high" was hyperintense with gray matter. ${ }^{12,17}$ If different signal intensities were detected in the parasellar areas, the lowest signal intensity was adopted. When the 2 neuroradiologists found different results, they discussed the case and came to a decision by consensus. We also reviewed all images of a series of follow-up MR images in patients who showed parasellar dark-signal-intensity areas on T2weighted images.

\section{Statistical Analysis}

Statistical analyses were performed by using StatView 5.0 software (SAS Institute, Cary, North Carolina). Between patients with LYH 

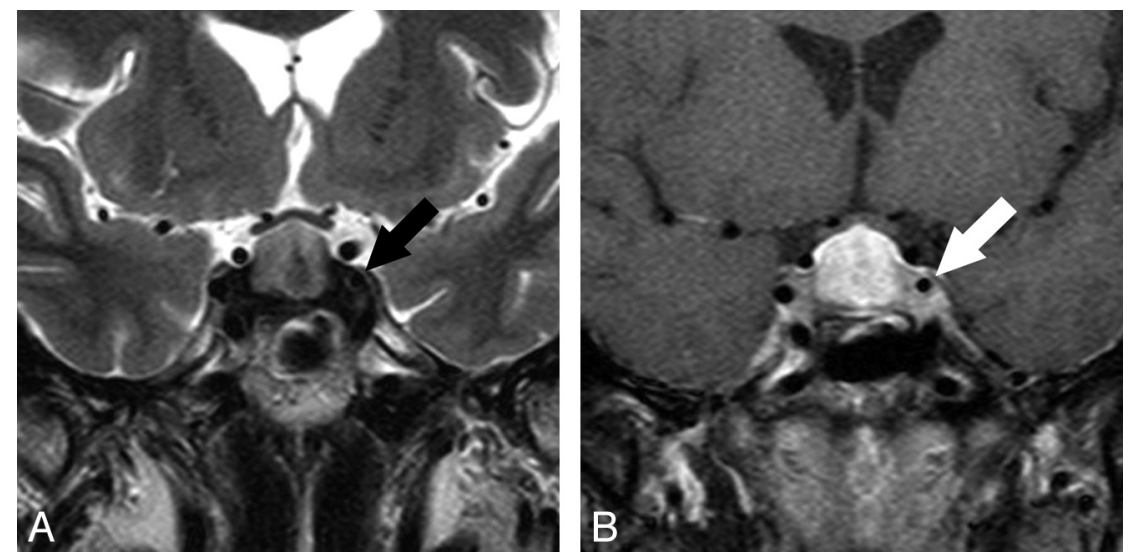

Fig 1. MR images of LYH (case 1) in a 38-year-old woman with partial hypopituitarism and diabetes insipidus. This patient had an 11-month history of headache, vomiting, polyuria, and polydipsia; transsphenoidal biopsy was performed and LYH proved histologically. $A$, Coronal T2-weighted image (TR/TE/NEX $=3500 / 120 \mathrm{~ms} / 3$ ) shows a large pituitary mass compressing the optic chiasm. A dark-signal-intensity area is seen in the cavernous sinus and sellar floor (arrow). B, Coronal contrast-enhanced T1-weighted image (TR/TE/NEX $=650 / 16$ ms/3) shows a large pituitary mass with homogeneous enhancement. Bilateral cavernous sinuses are swollen, with poor enhancement. The left ICA in the left cavernous sinus is narrowed (arrow).
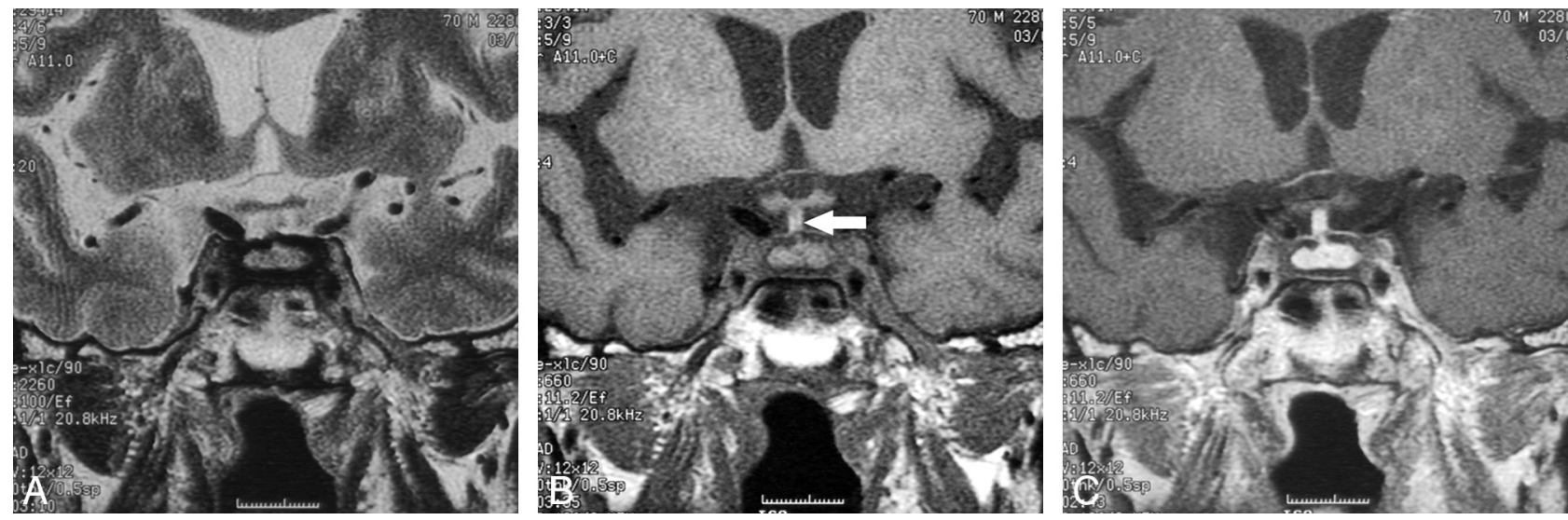

Fig 2. MR images of LYH (case 3) in a 70-year-old man with panhypopituitarism without diabetes insipidus. His chief complaint was general fatigue, and he was followed up for $>5$ years Replacement therapy (glucocorticoid, levothyroxine) was performed. A, Coronal T2-weighted image (TR/TE/NEX $=2260 / 100 \mathrm{~ms} / 3$ ) shows the relatively large pituitary gland. A dark-signal-intensity area is seen around the pituitary gland. The inner portions of the bilateral cavernous sinuses show low signal intensity. $B$, Coronal T1-weighted image (TR/TE/NEX $=$ $660 / 11.2 \mathrm{~ms} / 3$ ) shows the relatively large pituitary gland and stalk. A low-signal-intensity area is seen around the pituitary gland. The stalk shows high signal intensity, suggesting that it is storing antidiuretic hormone (arrow). C. Coronal contrast-enhanced T1-weighted image (TR/TE/NEX $=600 / 9.9$ ms/3) shows homogeneous enhancement in the pituitary gland and the stalk. The area around the pituitary gland and bilateral cavernous sinuses shows poor enhancement.

and those with pituitary adenoma, each imaging finding as described above was compared by using the Fisher exact probability test. $P$ values $<.05$ indicated statistical significance. We also calculated sensitivity and specificity for the diagnosis of LYH of each imaging finding as described above.

\section{Results}

Clinical findings for the 20 patients with LYH are summarized in On-line Table 1. Endocrinologic studies showed that 17 of 20 patients (85\%) had some degree of hypopituitarism: panhypopituitarism or partial hypopituitarism with diabetes insipidus in 12 patients, only panhypopituitarism or partial hypopituitarism in 2 patients, and only diabetes insipidus in 3 patients.

Clinical findings for the 22 patients with pituitary adenoma are summarized in On-line Table 2. Endocrinologic studies showed partial hyperpituitarism and partial hypopituitarism in 3 patients, only partial hyperpituitarism in 7 patients, and only hypopituitarism in 1 patient. In 4 patients who had hormonal examination of the posterior lobe, none had diabetes insipidus.
MR imaging findings of the patients with LYH and pituitary adenoma are summarized in On-line Table 3. PPHI was absent in 17 patients (85\%) with LYH and 3 (14\%) with pituitary adenoma. Ectopic PPHI was seen in 1 patient with LYH and 3 with pituitary adenoma. Seventeen patients (85\%) with LYH had thickened stalks. On the other hand, no patients $(0 \%)$ with pituitary adenoma had thickened stalks, though in 2 patients with LYH and 4 with pituitary adenoma, the stalk was unclear due to pituitary tumoral compression. The pituitary gland was symmetric in 17 patients (85\%) with LYH and in $2(9 \%)$ with pituitary adenoma. Homogeneous enhancement was seen in 13 patients $(65 \%)$ with LYH and in only 2 patients $(9 \%)$ with pituitary adenoma. The dural tail was seen in 13 patients $(65 \%)$ with LYH and 17 patients (77\%) with pituitary adenoma. On T2-weighted images, parasellar darksignal-intensity areas were seen in 7 patients (35\%) with LYH (Figs 1-3); the other 13 patients with LYH showed isointensity. Parasellar low-signal-intensity areas were seen in 2 patients (9\%) with pituitary adenoma (Fig 4), but no parasellar dark-signal-intensity areas were seen in any patients with pituitary adenoma. 

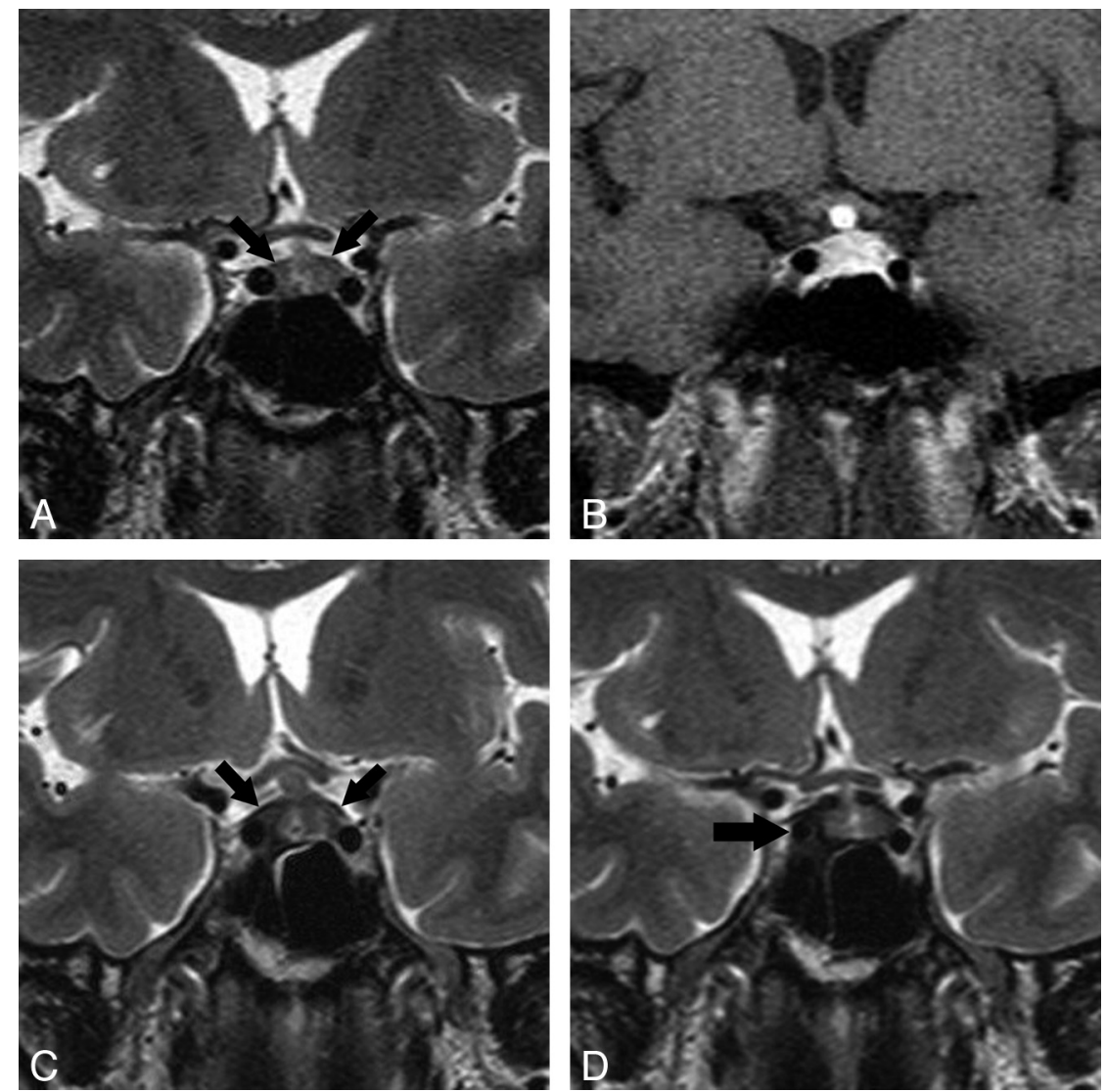

Fig 3. Serial MR images of LYH (case 4) in a 36-year-old man. Partial diabetes insipidus was observed in the early stage, and partial hypopituitarism was revealed later during the follow-up and treated by replacement therapy. $A$, The first coronal T2-weighted image (TR/TE/NEX $=3500 / 120 \mathrm{~ms} / 3$ ) shows the pituitary gland. Very thin dark-signal intensity areas on the upper edge of the pituitary gland (arrows) are seen. $B$, The first coronal contrast-enhanced T1-weighted image (TR/TE/NEX $=650 / 16 \mathrm{~ms} / 3$ ) shows a large pituitary gland with homogeneous enhancement. This section is slightly behind $A$. The large stalk with homogeneous enhancement slightly compresses the optic chiasm. $C$, The second coronal T2-weighted image (TR/TE/NEX $=3500 / 120 \mathrm{~ms} / 3$ ) 2 months after the first study shows a large pituitary gland and stalk. The previously noted thin dark-signal-intensity areas on the upper edge of the pituitary gland (arrows) were thicker than those in the first study. D, The third coronal T2-weighted image (TR/TE/NEX $=3500 / 120 \mathrm{~ms} / 3) 8$ months after the first study shows a large pituitary gland. The stalk is decreased in size. Dark-signal-intensity areas on the upper edge of the pituitary gland have thickened. The dark-signal-intensity area is enlarged and involves the right cavernous sinus; the right ICA in the cavernous sinus is narrower (arrow) than that in the previous study.

The other 20 patients with pituitary adenoma showed isointensity. On T1-weighted images, parasellar low-signalintensity areas were seen in 2 patients (10\%) with LYH. The other 18 patients with LYH showed isointensity. On the other hand, all patients with pituitary adenoma showed isointensity. Pituitary adenomas involved the cavernous sinuses in 11 patients; however, the cavernous sinuses were isointense with gray matter on T2- or T1-weighted images, and dark-signalintensity areas were not seen.

Details of the MR imaging findings in the 7 patients who showed parasellar dark signal intensity on T2-weighted images are summarized in On-line Table 4. Dark-signal-intensity areas in the cavernous sinuses were seen in 6 patients. Cavernous sinuses were swollen in 4 patients (Figs 1 and 2). Enhancement of the cavernous sinuses after an intravenous injection of contrast medium was poor in 3 patients (Figs 1 and 2). ICAs in the cavernous sinuses were narrowed in 3 patients (Figs 1 and 3 ). In cases 4,5 , and 6, dark-signal-intensity areas in the cavernous sinus were not seen in the first MR imaging study but appeared 2-20 months later. On T2-weighted images, darksignal-intensity bands around the anterior lobe and/or posterior lobe of the pituitary gland were seen in 4 patients (Fig 3). In case 5 , this band was not seen in the first MR imaging study but appeared 20 months later. In case 4, a very thin dark- signal-intensity band was noted on the upper edge of the posterior gland in the first study and was thickened on follow-up studies 2 and 8 months later (Fig 3).

Between patients with LYH and those with pituitary adenoma, a significant difference was identified by statistical analysis for the number of patients with PPHI on T1weighted images $(P<.0001)$, thickened stalk $(P<.0001)$, pituitary symmetry $(P<.0001)$, homogeneous enhancement $(P=.0001$; we excluded the cases without contrast enhancement), and dark-signal-intensity areas on T2weighted images $(P=.0015)$. No significant difference was identified for the number of patients with the dural tail ( $P=.36$; we excluded the cases without contrast enhancement) and low-signal-intensity areas on T1-weighted images $(P=.11)$. Sensitivity and specificity for the diagnosis for LYH were as follows: 0.85 and 0.95 for the loss of PPHI; 0.85 and 0.82 for the thickened stalk; 0.85 and 0.91 for the pituitary symmetry; 0.68 and 0.91 for the homogeneous enhancement (we excluded the cases without contrast enhancement); 0.65 and 0.23 for the dural tail; and 0.35 and 1.00 for the dark-signal-intensity areas on T2-weighted images, and 0.10 and 1.00 for the low-signal-intensity areas on T1-weighted images around the sellar and parasellar areas. 

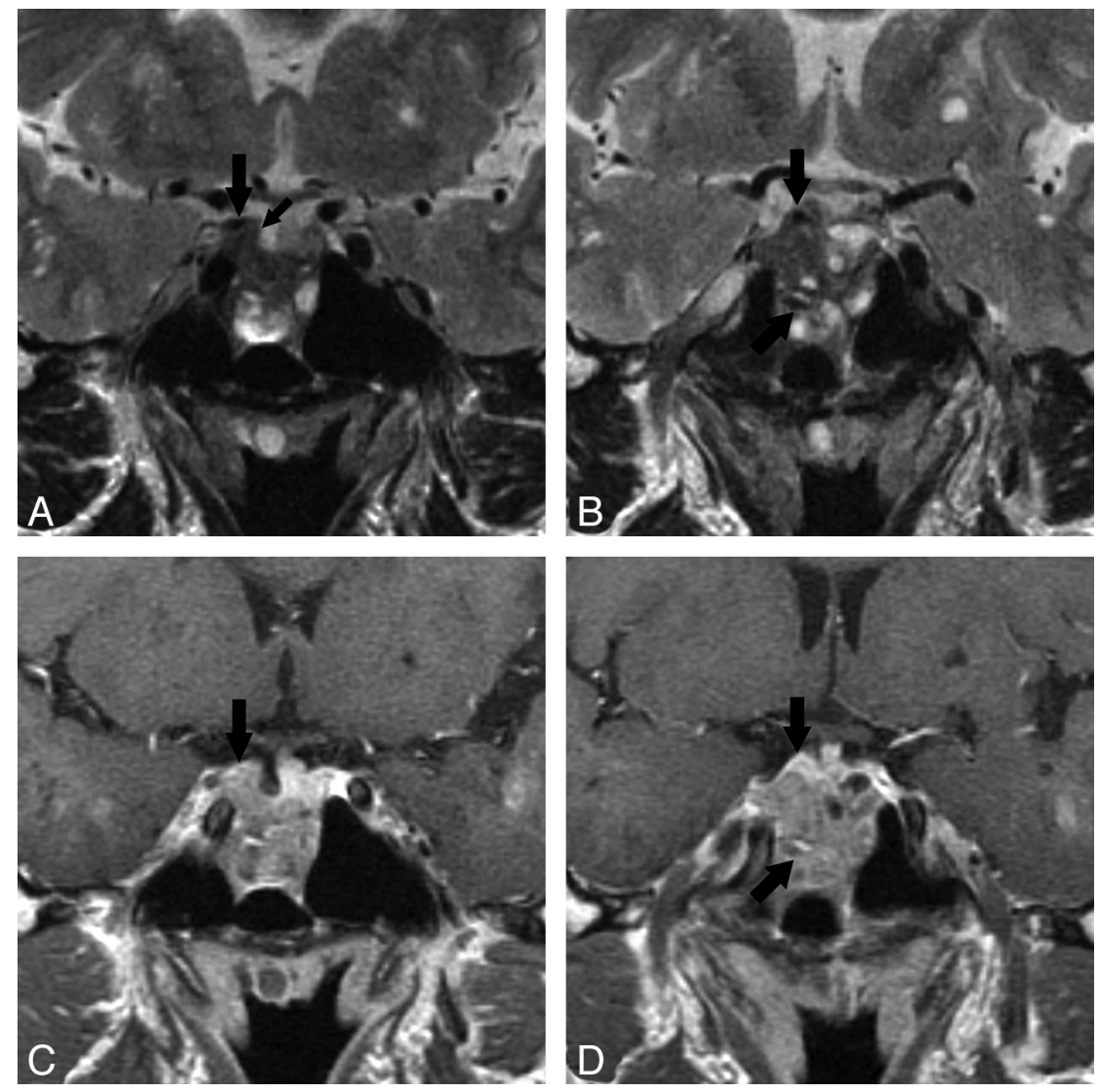

Fig 4. MR images of pituitary adenoma (case 5 ) in a 54-year-old woman. Acromegaly and partial hyperpituitarism were observed. She was treated with transsphenoidal resection, and the proved pathologic diagnosis was GH-secreting pituitary adenoma. $A$ and $B$, Coronal T2-weighted images (TR/TE/NEX $=3500 / 120$ ms/3) show a large pituitary mass destroying the sellar floor and extending in the sphenoid sinus. The upper portion of the mass is mixed low intensity, and the lower portion shows mixed high signals. Large arrows indicate small dark-signal-intensity areas in the pituitary mass. A low-signal-intensity margin is seen in the medial aspect of the upper pituitary mass (small arrow). $C$ and $D$, Coronal contrast-enhanced T1-weighted images (TR/TE/NEX $=600 / 9.9 \mathrm{~ms} / 3$ ) show heterogeneous enhancement in the pituitary mass. Arrows indicate enhanced focal areas in the pituitary mass, which correspond to the dark-signal-intensities on the T2-weighted images in $A$ and $B$.

\section{Discussion}

The present study reviewed a series of clinical and MR imaging findings in 20 patients with LYH. To the best of our knowledge, this study has reviewed original MR imaging findings in the largest number of patients with LYH. We demonstrated a new characteristic parasellar T2 dark sign (dark-signal-intensity area on T2-weighted images around the pituitary gland and in the cavernous sinus) in patients with LYH for the first time. Although sensitivity for the parasellar T2 dark sign was 0.35, lower than that for loss of PPHI, thickened stalk, pituitary symmetry, homogeneous enhancement, or the dural tail, specificity for the parasellar T2 dark sign was 1.00; and this finding was characteristic enough to distinguish with certainty the more common pituitary adenoma from LYH because false-positive cases were not found. T2-weighted images depicted the parasellar involvement of LYH well. Our results also suggest that there are variations of localization of T2 dark areas in LYH.

Some previous studies reported that loss of PPHI, thickened stalk, pituitary symmetry, homogeneous enhancement, and the dural tail were useful for distinguishing LYH from pituitary adenomas, ${ }^{2,3,8,11-14}$ but only 1 previous study investigated this statistically. Gutenberg et $\mathrm{al}^{14}$ reported that these findings contributed significantly to classifying the outcome as pituitary adenoma or autoimmune hypophysitis (including lymphocytic and granulomatous hypophysitis), and our results were consistent with their findings. ${ }^{14}$

Although MR imaging findings such as loss of PPHI, thickened stalk, pituitary symmetry, and homogeneous enhancement showed significant difference in the statistical analysis in the present study, these findings were nonspecific and were observed in both diseases. Loss of PPHI was observed in central diabetes insipidus due to idiopathic, inflammatory, or neoplastic processes, including pituitary adenoma. ${ }^{8,18-23}$ The stalk was sometimes unclear due to pituitary tumoral compression. Pituitary symmetry or asymmetry depends on tumor size, location, or invading areas. ${ }^{24}$ Cystic, necrotizing, or hemorrhagic changes also influence the enhancement pattern. ${ }^{25}$ Cystic appearance was described in $5 \%$ of the patients with LYH. ${ }^{1,8,11,12,26-34}$ Homogeneous enhancement can also be observed in pituitary adenomas. ${ }^{35}$ On the other hand, parasellar T2 dark sign was a characteristic finding in LYH and can contribute to distinguishing pituitary LYH from adenoma with certainty.

Clinical findings revealed that panhypopituitarism or partial hypopituitarism was seen in 17 patients (85\%) with LYH. On the other hand, partial hypopituitarism was seen in only 4 patients $(18 \%)$ with pituitary adenoma. We think that hypopituitarism may be useful clinical information, suggesting LYH rather than pituitary adenoma. The endocrinologic man- 
ifestation of LYH can vary widely, from only hyperprolactinemia, probably due to stalk compression, to panhypopituitarism mimicking Sheehan syndrome. ${ }^{1}$ In a recent report, the hormones most frequently impaired were ACTH, followed by TSH, LH/FSH, and vasopressin in patients with $\mathrm{LYH} .{ }^{8}$ On the other hand, hypogonadism rather than hypocortisolemia or diabetes insipidus is usually the initial problem in pituitary adenoma. ${ }^{4}$ In the present study, ACTH deficiency was seen in only 2 of 13 patients with LYH who had partial hypopituitarism, and insufficient hormones of the anterior pituitary lobe had some overlaps between pituitary adenoma and LYH. Isolated ACTH deficiency is not specific to LYH because it can be observed in the absence of $\mathrm{LYH},{ }^{36}$ and isolated deficiencies of other anterior pituitary hormones have been described in $\mathrm{LYH}^{37}$ We think that MR imaging findings, in addition to endocrinologic studies, can play an important role in distinguishing pituitary adenoma from LYH.

In the previous reports, histopathologic analysis revealed that LYH is extensively infiltrated by lymphoplasmacytic cells, consisting of lymphocytes, plasma cells, and macrophages. ${ }^{3}$ Lymphoplasmacytic aggregates surround the atrophic acini of pituitary cells, whereas the remaining pituitary tissue showed areas of reactive fibrosis. ${ }^{26,38}$ Caturegli et $\mathrm{al}^{8}$ reported that fibrosis was common (47\% of 267 cases of LYH) and often severe in their pathologic review. We think that dark-signalintensity areas on T2-weighted images around the pituitary gland and in the cavernous sinus reflected fibrotic changes accompanied by infiltration of lymphoplasmacytic cells. In the present study, the parasellar T2 dark signs were seen in the first MR imaging study in the 5 patients with LYH; however, this sign was not seen in the first MR imaging study of the 2 patients with LYH, but appeared 2-20 months after the first MR imaging study (On-line Table 4).

LYH is a chronic inflammatory disease that affects the pituitary gland and parasellar areas, and the duration of disease in patients with LYH is relatively long. ${ }^{3}$ We think that fibrotic changes progressed in the clinical course of LYH and appeared as parasellar T2 dark signs. We also think that the sensitivity and specificity of the parasellar T2 dark sign may vary with time, and thus the parasellar T2 dark sign may not be useful for making an initial diagnosis. If the parasellar T2 dark sign was not seen in the initial MR imaging in patients who were suspected of the diagnosis of LYH, further follow-up MR imaging studies may be needed to detect the sign.

In the present study, cases 1 and 2 were diagnosed histologically by transsphenoidal biopsy. In case 2, biopsy specimens showed adenohypophysitis with focal lymphocytic infiltrate with lymphoid follicle formation and attached portions of attenuated hyalinized fibrous tissue; however, fibrous tissue was not found in case 1 . We think the reason is that the biopsy specimens included only a small portion of the anterior lobes and did not include fibrous tissue. In the previous reports, approximately $10 \%$ of pituitary adenomas contained fibrosis. ${ }^{39}$ Iuchi et $\mathrm{al}^{40}$ reported that the signal intensities on $\mathrm{T} 2$ weighted images were significantly correlated with the percentage of collagen content of the pituitary adenomas, and adenomas that showed lower signal intensities on T2weighted images contained more collagen. However, low signal intensities on T2-weighted images in pituitary adenomas are usually seen in the pituitary masses and not outside the masses in the parasellar areas. However, the histopathologic findings of LYH were characterized by massive infiltration of lymphocytes and plasma cells followed by necrosis or surrounding parenchymal fibrosis. ${ }^{41}$ Therefore, the parasellar T2 dark sign is more suitable for LYH that infiltrates around the pituitary. Some previous studies have reported cavernous involvement in patients with $\mathrm{LYH},{ }^{5,6,10,42-46}$ but no studies have mentioned the parasellar T2 dark sign.

In the present study, we focused on the parasellar T2 dark sign in the differential diagnosis between LYH and pituitary adenoma; however, other differential diagnoses for parasellar T2 dark sign should be considered in practical clinical cases. Some cases such as sarcoidosis, lymphoma, or Tolosa-Hunt syndrome could potentially have relatively low signal intensity on T2-weighted images and may show findings similar to those of the parasellar T2 dark sign, though we have no experience of such cases. In particular, sarcoidosis should be carefully considered because it sometimes causes secondary granulomatous hypophysitis, ${ }^{4}$ and its differential diagnosis on MR imaging findings may be very difficult. We think that clinical presentation such as medical history and laboratory findings, in addition to MR imaging findings, needs to be investigated in detail in the diagnosis of LYH.

This study had several limitations. The first is that not all patients with LYH had pathologic confirmation; only 4 of 20 patients with LYH and only 2 of 7 patients with parasellar T2 dark sign had transsphenoidal biopsy. Additionally, there were 5 patients who had a history of autoimmune disease such as IgG4-related disorders, rheumatoid arthritis, and bullous pemphigoid. Other disorders, such as granulomatous hypophysitis or sarcoidosis, could feasibly have similar MR imaging findings. However, the duration of follow-up was relatively long (from 9 months to 9 years) in the 11 patients without pathologic confirmation or a history of autoimmune disease. Sarcoidosis is liable to recur not only in the intracranial area but also in the extracranial portion during longitudinal follow-up, but these 11 patients had no recurrence. In addition, they had no laboratory findings supporting sarcoidosis. Granulomatous hypophysitis is an extremely rare disorder (annual incidence of 1 in 10 million) and is usually diagnosed in postmortem specimens. ${ }^{4,47}$ The other limitation was that the present study was retrospective; most of the patients had only had a routine pituitary MR imaging study. If we had been able to prospectively evaluate these patients by using dynamic contrast-enhanced MR imaging ${ }^{5}$ or contrast-enhanced $3 \mathrm{D}$ constructive interference in the steady state, ${ }^{48}$ more details of the sellar and parasellar structures and signal intensity would have been shown. Further prospective studies that compare the findings obtained from high-field MR imaging systems or long-term follow-up MR imaging with the histopathologic findings should be conducted.

\section{Conclusions}

MR imaging findings, such as loss of PPHI, thickened stalk, pituitary symmetry, homogeneous enhancement, and parasellar T2 dark sign, can contribute to distinguishing pituitary adenoma from LYH. The parasellar T2 dark sign in particular was a characteristic finding in patients with $\mathrm{LYH}$, which was not observed in pituitary adenomas. 


\section{References}

1. Thodou E, Asa SL, Kontogeorgos G, et al. Lymphocytic hypophysitis: clinicopathological findings. J Clin Endocrinol Metab 1995;80:2302-11

2. Powrie JK, Powell M, Ayers AB, et al. Lymphocytic adenohypophysitis: magnetic resonance imaging features of two new cases and a review of the literature. Clin Endocrinol 1995;42:315-22

3. Bellastella A, Bizzarro A, Coronella C, et al. Lymphocytic hypophysitis: a rare or underestimated disease? Eur J Endocrinol 2003;149:363-76

4. Rivera JA. Lymphocytic hypophysitis: disease spectrum and approach to diagnosis and therapy. Pituitary 2006;9:35-45

5. Sato N, Sze G, Endo K. Hypophysitis: endocrinologic and dynamic MR findings. AJNR Am J Neuroradiol 1998;19:439-44

6. Supler ML, Mickle JP. Lymphocytic hypophysitis: report of a case in a man with cavernous sinus involvement. Surg Neurol 1992;37:472-76

7. Imura H, Nakao K, Shimatsu A, et al. Lymphocytic infundibuloneurohypophysitis as a cause of central diabetes insipidus. $N$ Engl $\mathrm{J} \mathrm{Med}$ 1993;329:683-89

8. Caturegli P, Newschaffer C, Olivi A, et al. Autoimmune hypophysitis. Endocr Rev 2005;26:599-614

9. Leung GK, Lopes MB, Thorner MO, et al. Primary hypophysitis: a singlecenter experience in 16 cases. J Neurosurg 2004;101:262-71

10. Nussbaum CE, Okawara S, Jacobs LS. Lymphocytic hypophysitis with involvement of the cavernous sinus and hypothalamus. Neurosurgery 1991;28:440-44

11. Saiwai S, Inoue Y, Ishihara T, et al. Lymphocytic adenohypophysitis: skull radiographs and MRI. Neuroradiology 1998;40:114-20

12. Ahmadi J, Meyers GS, Segall HD, et al. Lymphocytic adenohypophysitis: contrast-enhanced MR imaging in five cases. Radiology 1995;195:30-34

13. Chelaifa K, Bouzaidi K, Harzallah F, et al. Lymphocytic hypophysitis. J Neuroradiol 2002;29:57-60

14. Gutenberg A, Larsen J, Lupi I, et al. A radiologic score to distinguish autoimmune hypophysitis from nonsecreting pituitary adenoma preoperatively. AJNR Am J Neuroradiol 2009;30:1766-72

15. Kucharczyk W, Davis DO, Kelly WM, et al. Pituitary adenomas: high resolution MR imaging at 1.5 T. Radiology 1986;161:761-5

16. Leggett DA, Hill PT, Anderson RJ. 'Stalkitis' in a pregnant 32-year-old woman: a rare cause of diabetes insipidus. Australas Radiol 1999;43:104-07

17. Johnsen DE, Woodruff WW, Allen IS, et al. MR imaging of the sellar and juxtasellar regions. Radiographics 1991;11:727-58

18. Terano T, Seya A, Tamura Y, et al. Characteristics of the pituitary gland in elderly subjects from magnetic resonance imaging: relationship to pituitary hormone secretion. Clin Endocrinol (Oxf) 1996;45:273-79

19. Fujisawa I, Nishimura K, Asato R, et al. Posterior lobe of the pituitary in diabetes insipidus: MR findings. J Comput Assist Tomogr 1987;11:221-25

20. Sato N, Ishizaka $H$, Matsumoto $M$, et al. MR detectability of posterior pituitary high signal and direction of frequency encoding gradient. J Comput Assist Tomogr 1991;15:355-58

21. Sato N, Ishizaka H, Yagi H, et al. Posterior lobe of the pituitary in diabetes insipidus: dynamic MR imaging. Radiology 1993;186:357-60

22. Sato N, Endo K, Ishizaka H, et al. Serial MR intensity changes of the posterior pituitary in a patient with anorexia nervosa, high serum $\mathrm{ADH}$, and oliguria. J Comput Assist Tomogr 1993;17:648-50

23. Sato N, Endo K, Kawai H, et al. Hemodialysis: relationship between signal intensity of the posterior pituitary gland at MR imaging and level of plasma antidiuretic hormone. Radiology 1995;194:277-80

24. Chong BW, Kucharczyk W, Singer W, et al. Pituitary gland MR: a comparative study of healthy volunteers and patients with microadenomas. AJNR Am J Neuroradiol 1994;15:675-79
25. Bonneville JF, Bonneville F, Cattin F. Magnetic resonance imaging of pituitary adenomas. Eur Radiol 2005;15:543-48

26. Fehn M, Sommer C, Ludecke DK, et al. Lymphocytic hypophysitis: light and microscopic findings and correlation to clinical appearance. Endocr Pathol 1998;9:71-78

27. Sandler R, Danks KR, Hennigan $\mathrm{SH}$, et al. The widening spectrum of lymphocytic hypophysitis. J Ark Med Soc 1998;95:197-200

28. Ishihara $\mathrm{T}$, Hino $M$, Kurahachi $H$, et al. Long-term clinical course of two cases of lymphocytic adenohypophysitis. Endocr J 1996;43:433-40

29. McDermott MW, Griesdale DE, Berry K, et al. Lymphocytic adenohypophysitis. Can J Neurol Sci 1988;15:38-43

30. Farah JO, Rossi M, Foy PM, et al. Cystic lymphocytic hypophysitis, visual field defects and hypopituitarism. Int J Clin Pract 1999;53:643-44

31. Tamiya A, Saeki N, Kubota M, et al. Unusual MRI findings in lymphocytic hypophysitis with central diabetes insipidus. Neuroradiology 1999;41:899-900

32. Flanagan DE, Ibrahim AE, Ellison DW, et al. Inflammatory hypophysitis: the spectrum of disease. Acta Neurochir (Wien) 2002;144:47-56

33. Lee SJ, Yoo HJ, Park SW, et al. A case of cystic lymphocytic hypophysitis with cacosmia and hypopituitarism. Endocr J 2004;51:375-80

34. Pérez-Núñez A, Miranda P, Arrese I, et al. Lymphocytic hypophysitis with cystic MRI appearance. Acta Neurochir (Wien) 2005;147:1297-300

35. Heshmati HM, Kujas M, Casanova S, et al. Prevalence of lymphocytic infiltrate in $\mathbf{1 4 0 0}$ pituitary adenomas. Endocr J 1998;45:357-61

36. Nagai Y, Ieki Y, Ohsawa K, et al. Simultaneously found transient hypothyroidism due to Hashimoto's thyroiditis, autoimmune hepatitis and isolated ACTH deficiency after cessation of glucocorticoid administration. Endocr J 1997;44:453-8

37. Barkan AL, Kelch RP, Marshall JC. Isolated gonadotrope failure in the polyglandular autoimmune syndrome. N Engl J Med 1985;312:1535-40

38. Durán Martinez M, Santonja C, Pavon de Paz I, et al. Lymphocytic hypophysitis: report of an unusual case of a rare disorder. J Endocrinol Invest 2001;24:190-93

39. Wang H, Li WS, Shi DJ, et al. Correlation of MMP(1) and TIMP (1) expression with pituitary adenoma fibrosis. J Neurooncol 2008;90:151-56

40. Iuchi T, Saeki N, Tanaka M, et al. MRI prediction of fibrous pituitary adenomas. Acta Neurochir (Wien) 1998;140:779-86

41. Nishioka H, Ito H, Miki T, et al. A case of lymphocytic hypophysitis with massive fibrosis and the role of surgical intervention. Surg Neurol 1994;42:74-78

42. Kartal I, Yarman S, Tanakol R, et al. Lymphocytic panhypophysitis in a young man with involvement of the cavernous sinus and clivus. Pituitary 2007;10:75-80

43. Melgar MA, Mariwalla N, Gloss DS, et al. Recurrent lymphocytic hypophysitis and bilateral intracavernous carotid artery occlusion: an observation and review of the literature. Neurol Res 2006;28:177-83

44. Lecube A, Francisco G, Rodríguez D, et al. Lymphocytic hypophysitis successfully treated with azathioprine: first case report. J Neurol Neurosurg Psychiatry 2003;74:1581-83

45. Tubridy N, Saunders D, Thom M, et al. Infundibulohypophysitis in a man presenting with diabetes insipidus and cavernous sinus involvement. J Neurol Neurosurg Psychiatry 2001;71:798-801

46. Nakamura Y, Okada H, Wada Y, et al. Lymphocytic hypophysitis: its expanding features. J Endocrinol Invest 2001;24:262-67

47. Cheung CC, Ezzat S, Smyth HS, et al. The spectrum and significance of primary hypophysitis. J Clin Endocrinol Metab 2001;86:1048-53

48. Yagi A, Sato N, Taketomi A, et al. Normal cranial nerves in the cavernous sinuses: contrast-enhanced three-dimensional constructive interference in the steady state MR imaging. AJNR Am J Neuroradiol 2005;26:946-50 